\title{
Feasibility of Dried Blood Spots in Children with Behavioral Problems
}

\author{
Sanne M. Kloosterboer, MD, * Estelle van Eijk, BSc, * Monique van Dijk, PhD, $\neq$ \\ Gwen C. Dieleman, MD, PhD, † Manon H. J. Hillegers, MD, PhD, † Teun van Gelder, MD, PhD,* \\ Birgit C. Koch, PharmD, PhD,* and Bram Dierckx, MD, PhD $†$
}

\begin{abstract}
Background: Minimally invasive sampling methods are important to facilitate therapeutic drug monitoring and pharmacokinetic research in children with behavioral problems. This study assessed the feasibility and pain of dried blood spot (DBS) sampling in this population.
\end{abstract}

Methods: Repeated DBS sampling was performed in children with autism spectrum disorder (ASD) and severe behavioral problems using antipsychotic drugs, aged between 6 and 18 years. The child, guardian, and DBS performer assessed pain using the numeric rating scale (NRS-11) or 5-face Faces Pain Scale. The influence of age, sex, and the fingerprick performer on the child's pain intensity was analyzed using linear mixed models.

Results: Overall, 247 fingerpricks were performed in 70 children. Seven children refused all DBS sampling. The median (interquartile range) NRS-11 pain scores were 2 (3) rated by children, 3 (2.5) by guardians, and 2 (2) by fingerprick performers. The child's age and sex, and fingerprick performer had no significant influence on pain intensity.

Conclusions: DBS sampling could be performed in most children with ASD and severe behavioral problems. However, 1 in 5 children refused one or more DBS fingerpricks owing to distress. Most expressed minimal pain (NRS $<4$ ). Repeated sampling with DBS is feasible in children with ASD and severe behavioral problems.

Key Words: dried blood spot, children, adolescents, autism spectrum disorder, behavioral problems

(Ther Drug Monit 2020;42:648-651)

Received for publication January 8, 2020; accepted May 6, 2020.

From the Departments of *Hospital Pharmacy, and †Child and Adolescent Psychiatry/Psychology, Erasmus MC, University Medical Center Rotterdam; and \$Departments of Pediatric Surgery and Internal Medicine, Section Nursing Science, Erasmus MC, University Medical Center Rotterdam, the Netherlands.

S. M. Kloosterboer, B. Dierckx, and B. C. Koch received grant research support from The Netherlands Organization for Health Research and Development (ZonMW), number 836041011. T. van Gelder has received honoraria for consulting and/or presentations from Astellas, Aurinia, Vitaeris, and Roche Diagnostics. The remaining authors declare no conflict of interest.

Correspondence: Sanne M. Kloosterboer, MD, Department of Hospital Pharmacy, Erasmus MC, University Medical Center Rotterdam, Dr. Molewaterplein 40, 3015 GD Rotterdam, The Netherlands (e-mail: s. kloosterboer@erasmusmc.nl).

Copyright (C) 2020 Wolters Kluwer Health, Inc. All rights reserved.

\section{INTRODUCTION}

As an alternative to invasive venipuncture, dried blood spot (DBS) analysis is well established for drug quantification. ${ }^{1}$ DBS involves only one fingerprick and is especially suited for pediatric populations, in clinical and research settings. DBS can increase sampling tolerability particularly in children with behavioral problems. In these children, blood sampling by venipuncture is often challenging due to restlessness or aggression; the minimally invasive DBS procedure could overcome these challenges and can be performed at home.

Notably, among children with behavioral problems, DBS sampling is of particular interest in children prescribed antipsychotic drugs. These drugs are effective in a wide range of psychiatric disorders in childhood, ${ }^{2}$ including behavioral problems in autism spectrum disorder (ASD); however, these drugs are associated with serious side effects. The most important side effect concerns weight gain, ${ }^{3}$ leading to metabolic abnormalities, ${ }^{3}$ diabetes mellitus, ${ }^{4}$ cardiovascular diseases, ${ }^{5}$ and possibly even unexpected death ${ }^{6}$ in children using antipsychotic drugs. Therapeutic drug monitoring (TDM) could be an important tool to increase the safety of these drugs in children. ${ }^{7,8}$

To facilitate TDM research and applicability in children using antipsychotic drugs, a DBS assay for the quantification of risperidone, aripiprazole, and pipamperone has been developed..$^{9,10}$ However, the feasibility and burden of DBS in children with severe behavioral problems remains unexplored. In this study, we evaluated the feasibility and pain levels of DBS sampling in children with ASD and severe behavioral problems and investigated the influence of sex, age, and fingerprick performer on the child's pain intensity.

\section{MATERIALS AND METHODS}

\section{Study Population}

Children, aged 6-18 years, with ASD according to the Diagnostic and Statistical Manual of Mental Disorders IV ${ }^{11}$ or $5^{12}$ and severe behavioral problems were included in an observational study investigating the relationship between antipsychotic drug concentrations and effects (the Netherlands National Trial Register NTR6050). All included children were prescribed risperidone, aripiprazole, or pipamperone, the 3 most commonly used antipsychotic drugs in the Netherlands. ${ }^{13}$ This study, including the pain assessments, was approved by the medical ethics committee of the Erasmus MC (MEC 2016-124). All patients and/or their legal 
representatives provided written informed consent before entering the study.

\section{DBS Sampling}

Each child received 2 to 3 fingerpricks on 2 separate days, with a minimum of 1 hour between 2 fingerpricks to allow random sampling. The fingerpricks were performed with a single-use contact-activated lancet (BD Microtainer 2.0 $\times 1.5 \mathrm{~mm}$ ), with the first performed by the research staff at the clinic. Subsequent fingerpricks were performed by research staff, the guardian, or the child itself, at the clinic or home depending on the patient's preference. Most children underwent a venipuncture the same day. Antipsychotic drug concentrations were determined using a previously validated ultra-high performance liquid chromatography-mass spectrometry method for $\mathrm{DBS}^{9,10}$

\section{Pain Assessment}

Before performing the fingerprick, the child's likely response to procedures, coping strategies, and possible interventions were discussed with parents and the child. The DBS procedure was explained and demonstrated before the performance. Immediately after the fingerprick, the child, guardian, and fingerprick performer assessed pain. Children aged 6 years used a 5-face Faces Pain Scale; older children, guardians, and fingerprick performers used the 11-point numeric rating scale (NRS-11). ${ }^{14}$ The Faces Pain Scale demonstrates 5 faces from happy to extremely sad. For children with cognitive impairment, the Checklist Pain Behavior (CPG), ${ }^{15}$ including the NRS-11, was scored by the research staff, whereas the guardians only scored the NRS-11. An NRS score of 4 or higher and a CPG score of 5 or higher is regarded as an indication for pain-relieving interventions. ${ }^{15,16}$ After each fingerprick, the researcher evaluated the burden for each child based on pain scores, assessing whether a subsequent fingerprick could be performed together with the child and the guardian.

\section{Statistical Analyses}

Groups were compared using the Mann-Whitney $U$ test for continuous data (nonnormal data distribution) and the Fisher exact test for categorical data. The interrater reliability of pain scores rated by the child, guardian, and research staff was analyzed with the intraclass correlation coefficient using a 2-way mixed model with absolute agreement type. To evaluate the influence of the child's age and sex, as well as fingerprick performer, on the child's pain, a linear mixed model analysis was used with a random intercept. Sampling days were clustered within patients. All variables were tested using a univariate model. Variables with $P<0.10$ were added to the multivariate model; variables with $P<0.05$ in this model were selected for the final model. All analyses were performed using SPSS version 25 (SPSS Inc, Chicago, IL).

\section{RESULTS}

Overall, 81 children were eligible for inclusion. Seven children refused all DBS sampling (8.6\%), and in 9 children
(11.1\%) not all DBS sampling could be performed as they expected DBS to be extremely painful or stressful (Fig. 1). Children who refused one or more DBS samples were significantly younger than those who performed all DBS samples (median age 9.3 versus 10.8 years, $P=0.43$ ). The groups did not significantly differ in terms of sex or mental retardation.

Totally, 253 DBS samples were collected from 70 children, with 21 female $(30 \%)$ children, and a median [interquartile range (IQR)] age of 10.7 (5.0) years. Twelve children were diagnosed with cognitive impairment. Most DBS fingerpricks were performed by research staff $(63.0 \%)$, followed by guardians $(36.6 \%)$ and the child $(0.4 \%)$. DBS samples obtained by guardians were performed at home. Notably, 39 (15.4\%) DBS samples were not of sufficient quality for analysis.

For 247 DBS samples, 1 or more NRS-11 pain scores were available, including 168 pain scores reported by the child, 187 by guardians, and 126 by research staff. The median (IQR) NRS-11 pain scores were 2 (3) rated by children, 3 (2.5) by guardians, and 2 (2) by research staff. The medians and ranges are shown in Figure 2. Furthermore, 61 fingerpricks $(36.3 \%)$ were rated $\geq 4$ by the child. Twenty-six CPG scores were available; the median (IQR) score was 3 (1) and $11.5 \%$ were 5 or higher.

For 147 fingerpricks, both child and guardian NRS-11 pain scores were available. The intraclass correlation coefficient for guardians and children was 0.86 (95\% CI 0.81-0.90) and for research staff and children was 0.79 (95\% CI $0.67-$ 0.86), considered excellent and good, respectively.

For 167 NRS-11 scores issued by the child, the age and sex of the child, as well as the nature of fingerprick performer, were known. The univariate mixed model analysis showed that none of these variables significantly influenced the child's pain intensity as follows: age of the child $(\beta=-0.087, P=0.134)$, sex of the child $(\beta=0.476$, $P=0.225$ ), and fingerpick performer (guardian $\beta=0.266$, $P=0.211$, self $\beta=1.178, P=0.548$, with research staff as reference category).

\section{DISCUSSION}

Repeated DBS sampling was successfully performed in most children with ASD and severe behavioral problems. Nevertheless, 1 in 5 children refused one or more DBS fingerpricks due to distress. However, most children expressed minimal pain (NRS $<4$ ) during DBS sampling.

Relatively little is known regarding pain in autistic children, although hyperreactivity or hyporeactivity to sensory input is a feature of ASD. ${ }^{12}$ Comparable, reduced, and increased pain thresholds have been reported in the literature. ${ }^{17}$ This is consistent with our study, where both extremely low and extremely high pain scores were observed, although most pain scores were lower than the generally accepted threshold of 4 . However, pain assessment in children with ASD is challenging because they may express pain differently due to the lack of social responsiveness and language impairment. ${ }^{18}$ Unfortunately, pain assessment tools specifically for individuals with ASD have remained largely unexplored. 


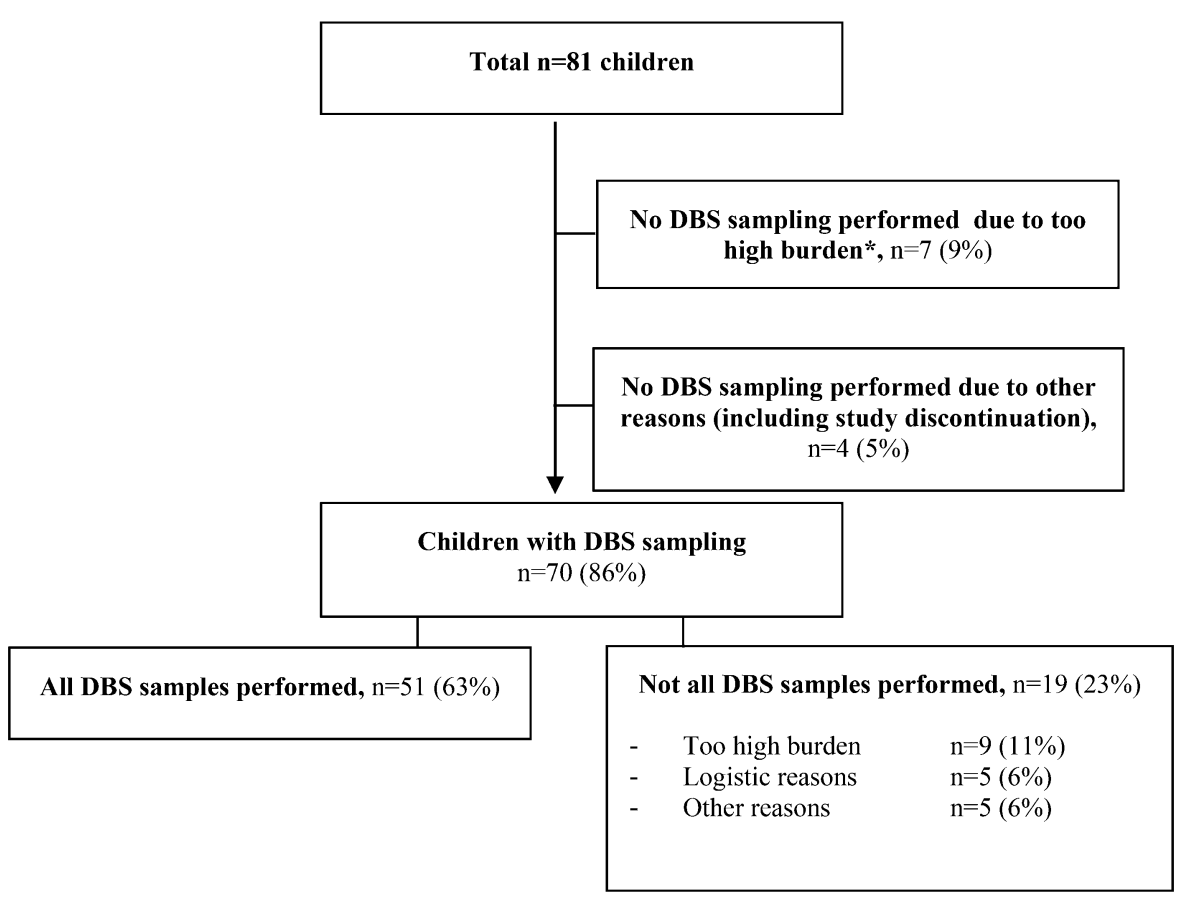

Figure 1. DBS sampling fulfillment.

* These children expected DBS to be too painful or stressful

The tolerability of blood sampling is not only determined by pain but also by distress and anxiety. Children with ASD express higher levels of distress during venipuncture than their nonimpaired peers. ${ }^{19,20}$ DBS sampling may reduce many of these stressors because the sampling preparation phase is considerably less complex, allowing sampling by a guardian in the home setting. Hence, most children with high levels of distress are expected to prefer DBS sampling over venipuncture, as do their normally developing peers, ${ }^{21,22}$ but further research is necessary for validation.

In children with behavioral problems, the performance of repeated sampling studies is highly challenging. As previously suggested, sparse sampling designs in combination with DBS sampling can facilitate pediatric pharmacokinetic research..$^{23,24}$ Both the lower burden and acceptance of home sampling improve study recruitment, which is particularly difficult in child and adolescent psychiatry. However, clinical validation remains challenging, for example, our study showed a larger variability in antipsychotic drug concentrations measured with DBS than with venipuncture. As advanced pharmacokinetic modeling techniques allow the correction of this variability, the advantages of DBS can still outweigh the disadvantages. Currently, this method remains primarily useful in research settings; however, further development and study of DBS and TDM could yield a clinical, minimally invasive tool that improves the safety of antipsychotic drugs.

This study has some limitations. As mentioned earlier, self-reporting of pain might be unreliable in children with ASD, leading to an overestimation or underestimation of pain. However, no pain measurement tools are currently available for pain assessment specifically in children with ASD. To optimize pain assessment, it was ensured that the DBS sampling performers had previous experience in communicating with children with ASD and that the child's guardians were present. Previous research has shown that parent involvement is essential for the interpretation and expression of pain in children with ASD. ${ }^{18}$ A comparison with the pain associated with conventional venipuncture was not conducted, limiting the evaluation of the most optimal sampling technique in this population.

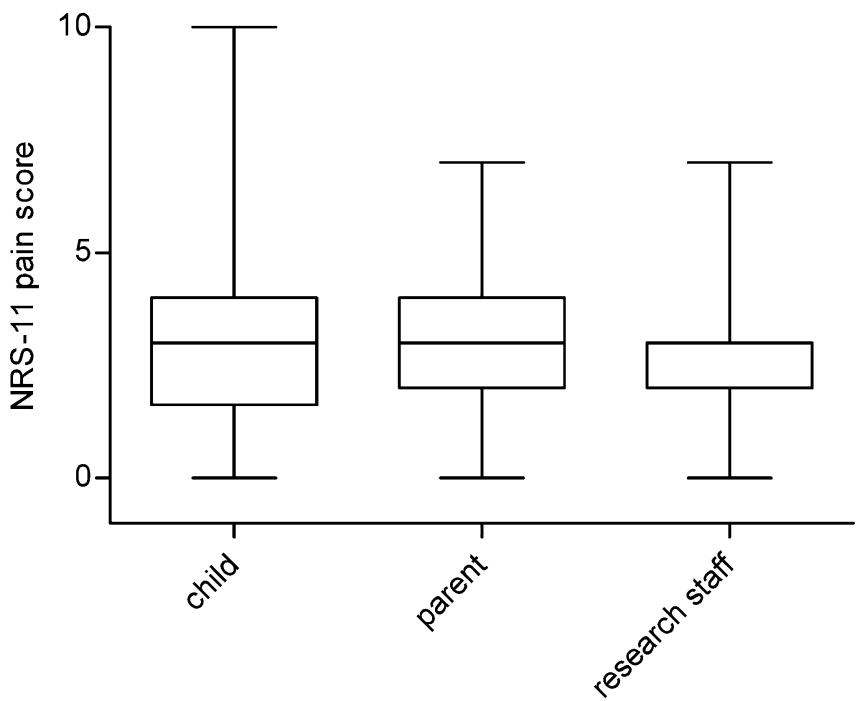

FIGURE 2. Child's pain associated with DBS sampling rated by child, guardian, and research staff. Whiskers indicate minimum-maximum. . 


\section{CONCLUSIONS}

This is the first study that evaluated the feasibility of repeated DBS sampling in children with ASD and severe behavioral problems, indicating that DBS is a feasible sampling technique in this population. Although further research is needed to compare the burden of DBS and venipuncture in children with ASD and behavioral problems, DBS can facilitate pharmacokinetic research and TDM in these and other pediatric populations where sampling remains challenging.

\section{REFERENCES}

1. Sharma A, Jaiswal S, Shukla M, et al. Dried blood spots: concepts, present status, and future perspectives in bioanalysis. Drug Test Anal. 2014;6:399-414.

2. Lee ES, Vidal C, Findling RL. A focused review on the treatment of pediatric patients with atypical antipsychotics. $J$ Child Adolesc Psychopharmacol. 2018;28:582-605.

3. Cohen D, Bonnot O, Bodeau N, et al. Adverse effects of secondgeneration antipsychotics in children and adolescents: a Bayesian metaanalysis. J Clin Psychopharmacol. 2012;32:309-316.

4. Bobo WV, Cooper WO, Stein CM, et al. Antipsychotics and the risk of type 2 diabetes mellitus in children and youth. JAMA Psychiatry. 2013; 70:1067-1075.

5. McIntyre RS, Jerrell JM. Metabolic and cardiovascular adverse events associated with antipsychotic treatment in children and adolescents. Arch Pediatr Adolesc Med. 2008;162:929-935.

6. Ray WA, Chung CP, Murray KT, et al. Atypical antipsychotic drugs and the risk of sudden cardiac death. $N$ Engl J Med. 2009;360:225-235.

7. Gerlach M, Egberts K, Dang SY, et al. Therapeutic drug monitoring as a measure of proactive pharmacovigilance in child and adolescent psychiatry. Expert Opin Drug Saf. 2016;15:1477-1482.

8. Egberts KM, Mehler-Wex C, Gerlach M. Therapeutic drug monitoring in child and adolescent psychiatry. Pharmacopsychiatry. 2011;44:249-253.

9. Tron C, Kloosterboer SM, van der Nagel BCH, et al. Dried blood spots combined with ultra-high-performance liquid chromatography-mass spectrometry for the quantification of the antipsychotics risperidone, aripiprazole, pipamperone, and their major metabolites. Ther Drug Monit. 2017;39:429-440.
10. Kloosterboer SM, de Winter BCM, Bahmany S, et al. Dried blood spot analysis for therapeutic drug monitoring of antipsychotics: drawbacks of its clinical application. Ther Drug Monit. 2018;40:344-350.

11. American Psychiatric Association. Diagnostic and Statistical Manual of Mental Disorders. 4th ed. Washington, DC: American Psychiatric Association; 2000.

12. American Psychiatric Association. Diagnostic and Statistical Manual of Mental Disorders. 5th ed. Washington, DC: American Psychiatric Association; 2013.

13. Kloosterboer SM, Schuiling-Veninga CCM, Bos JHJ, et al. Antipsychotics in Dutch youth: prevalence, dosages, and duration of use from 2005 to 2015. J Child Adolesc Psychopharmacol. 2018;28:173-179.

14. Castarlenas E, Jensen MP, von Baeyer CL, et al. Psychometric properties of the numerical rating scale to assess self-reported pain intensity in children and adolescents: a systematic review. Clin J Pain 2017;33: 376-383.

15. Duivenvoorden HJ, Tibboel D, Koot HM, et al. Pain assessment in profound cognitive impaired children using the checklist pain behavior; is item reduction valid? Pain. 2006;126:147-154.

16. McCaffery MPC. Pain Clinical Manual. 2nd ed. St. Louis, MO: V. Mosby; 1999.

17. Moore DJ. Acute pain experience in individuals with autism spectrum disorders: a review. Autism. 2015;19:387-399.

18. Ely E, Chen-Lim ML, Carpenter KM II, et al. Pain assessment of children with autism spectrum disorders. J Dev Behav Pediatr. 2016; 37:53-61.

19. Nader R, Oberlander TF, Chambers CT, et al. Expression of pain in children with autism. Clin J Pain. 2004;20:88-97.

20. Rattaz C, Dubois A, Michelon C, et al. How do children with autism spectrum disorders express pain? A comparison with developmentally delayed and typically developing children. Pain. 2013;154:2007-2013.

21. Al-Uzri A, Freeman KA, Wade J, et al. Longitudinal study on the use of dried blood spots for home monitoring in children after kidney transplantation. Pediatr Transpl. 2017;21. Pubmed ID 28635157.

22. Verougstraete N, Lapauw B, Van Aken S, et al. Volumetric absorptive microsampling at home as an alternative tool for the monitoring of hbalc in diabetes patients. Clin Chem Lab Med. 2017;55:462-469.

23. Patel P, Mulla H, Tanna S, et al. Facilitating pharmacokinetic studies in children: a new use of dried blood spots. Arch Dis Child. 2010;95:484487.

24. Barker CIS, Standing JF, Kelly LE, et al. Pharmacokinetic studies in children: recommendations for practice and research. Arch Dis Child. 2018;103:695-702. 